\title{
Editorial: The Physics of Diamond and its Technological Applications
}

\author{
Silvio Sciortino ${ }^{1,2 *}$ and Tzveta Apostolova $a^{3,4}$ \\ ${ }^{1}$ Department of Physics and Astronomy, University of Florence, Florence, Italy, ${ }^{2}$ National Institute for Nuclear Physics (INFN), \\ Florence, Italy, ${ }^{3}$ Institute for Nuclear Research and Nuclear Energy (BAS), Sofia, Bulgaria, ${ }^{4}$ Institute for Advanced Physical Studies, \\ New Bulgarian University, Sofia, Bulgaria
}

Keywords: diamond, radiation sensors and dosimeters, quantum technologies, ultrafast laser irradiation, timing measurements, theoretical modeling, diamond devices, ion implantation

Editorial on the Research Topic

The Physics of Diamond and its Technological Applications

Synthesis of diamond by Chemical Vapour Deposition (CVD) was achieved already in the 60s, but applications became viable only in the 80-90s due to a significant improvement of the technique in which the substrate temperature was decoupled from the gas phase temperature leading to an increase of orders of magnitude in growth rates [1]. The CVD technique offered a superior control of the purity of the material with respect to natural and High-Pressure High-Temperature synthetic diamond, in particular limiting to atomic ppb the unwanted inclusion of substitutional $N$ and $N$ aggregates which severely deteriorate the electronic properties of the material. A huge industrial scale-up toward high technology applications was forecasted to happen in the 2000s [2], thanks to the extreme physical properties of the material, suitable for countless applications. Yet, that revolution did not happen and the field is still strongly limited by the polycrystalline nature of the material growth on non-diamond substrates, which severely impairs the electronics applications. Applications of homoepitaxially grown monocrystalline material is also strongly limited because the material itself can be fabricated with a maximum size of about $1 \mathrm{~cm}^{2}$, due the availability of diamond substrates and by the cost of the production. In addition, monocrystalline samples are also affected by structural defects, such as dislocations, that degrade their optical and electronic response. Heteroepitaxial growth of large area monocrystalline samples has been recently reported [3]. Although the electronic properties of this material are still lower quality than those of the homoepitaxial one [4], optimization is underway. Heteroepitaxial material seems the only viable solution for a future widespread development of diamond applications. Nevertheless, many interesting applications of CVD diamond are already possible at present and others that offer the promise of a major breakthrough, are intensively investigated. Among the latter, the concept of diamond power electronics, although still limited by the considerable depth of the dopant $(\mathrm{P}, \mathrm{B})$ levels [5], is extremely promising. Particularly appealing is the development of fast electronics operated without need of cooling and offering the possibility of integrating diamond sensors with diamond readout electronics in compact and extremely radiation tolerant devices.

In this Topic we address some of the CVD diamond applications which seem more mature. Diamond sensors for high resolution timing application have been used (Minafra et al.) in high energy experiments to reconstruct particle trajectories and measure their arrival time, profiting of the radiation hardness and the high saturation velocities of the material. A three-dimensional electrode architecture has been proposed within the TimeSpot experiment both for diamond and silicon particle detectors where the drift path of the ionization carriers towards the electrodes is strongly shortened. In this way the influence of the crystal defects as well as the charge collection times are substantially reduced resulting in some of the fastest [5] and radiation hardest [6] radiation sensors ever fabricated. Anderlini et al. summarize the results obtained so far on 3D diamond detectors 
within the framework of TimeSpot. A quantitatively direct comparison of $3 \mathrm{D}$ silicon and diamond radiation hardness in the very same environment has not yet been presented and this would be a major step forward to assess the domain of application of diamond 3D sensors.

Diamond is also the best material for radiation dosimetry in radiotherapy due to its water equivalence. CVD diamond has replaced natural diamond as fundamental material for commercial dosimeters. Talamonti et al. resume the state of the art of CVD diamond clinical dosimeters. Three-D diamond pixel dosimeters can provide the very high resolution needed in real time small field dosimetry.

A deep insight into the fundamental physical processes in diamond irradiated with intense, ultrashort laser pulses is of paramount importance to optimize the fabrication of diamond sensors and dosimeters. A review of the present state of the art of experimental and theoretical studies on this topic is presented by Apostolova et al. The work also gives a comprehensive picture of

\section{REFERENCES}

1. Bachmann PK. Microwave Plasma CVD and Related Techniques for Low Pressure diamond Synthesis. In: A Lettington and J Steeds, editors. Thin Film diamond. London: Chapman \& Hall (1994).

2. K Spear and J Dismukes, Editors. Synthetic Diamond - Emerging CVD Science and Technology. New York: Wiley (1994).

3. Schreck M, Gsell S, Brescia R, and Fischer M. Ion Bombardment Induced Buried Lateral Growth: the Key Mechanism for the Synthesis of Single crystal diamond Wafers. Sci Rep (2017) 7:44462. doi:10.1038/srep44462

4. Berdermann E, Afanaciev K, Ciobanu M, Fischer M, Gsell S, Kiš M, et al. Progress in Detector Properties of Heteroepitaxial diamond Grown by Chemical Vapor Deposition on Ir/YSZ/Si(001) Wafers. Diamond Relat Mater (2019) 97:107420. doi:10.1016/ j.diamond.2019.05.006

5. Lai A, Anderlini L, Aresti M, Bizzeti A, Cardini A, Betta GFD, et al. First Results of the TIMESPOT Project on Developments on Fast Sensors for Future Vertex Detectors. Nucl Instr Methods Phys Res Section A: Acc Spectrometers, Detectors Associated Equipment (2020) 981:164491. doi:10.1016/j.nima.2020.164491 the experimental laser technology used for innovative diamond devices.

Due to its large bandgap, diamond can host a number of interbandgap states which can be tailored for quantum technologies. These can be color centers for single-photon emission (Lagomarsino et al.), temperature or ultra-weak magnetic field sensors (Fabbri et al.). Quantum control and the capability of full deterministic implantation/activation of single emitters are the issues to be addressed.

All these applications which are within reach in the near future, will undoubtedly benefit of the continuous progress of CVD diamond synthesis.

\section{AUTHOR CONTRIBUTIONS}

All authors listed have made a substantial, direct, and intellectual contribution to the work, and approved it for publication.

6. Lagomarsino S, Bellini M, Corsi C, Cindro V, Kanxheri K, and Morozzi A. Radiation Hardness of Three-Dimensional Polycrystalline diamond Detectors. Appl Phys Lett (2015) 106:193509. doi:10.1063/1.4921116

Conflict of Interest: The authors declare that the research was conducted in the absence of any commercial or financial relationships that could be construed as a potential conflict of interest.

Publisher's Note: All claims expressed in this article are solely those of the authors and do not necessarily represent those of their affiliated organizations, or those of the publisher, the editors and the reviewers. Any product that may be evaluated in this article, or claim that may be made by its manufacturer, is not guaranteed or endorsed by the publisher.

Copyright (c) 2021 Sciortino and Apostolova. This is an open-access article distributed under the terms of the Creative Commons Attribution License (CC $B Y)$. The use, distribution or reproduction in other forums is permitted, provided the original author(s) and the copyright owner(s) are credited and that the original publication in this journal is cited, in accordance with accepted academic practice. No use, distribution or reproduction is permitted which does not comply with these terms. 